\title{
On a composition of two Hilbert-Hardy-type integral operators and related inequalities
}

Bicheng Yang ${ }^{1 *}$ and Qiang Chen ${ }^{2}$

\author{
"Correspondence: \\ bcyang@gdei.edu.cn; \\ bcyang818@163.com \\ 1 Department of Mathematics, \\ Guangdong University of \\ Education, Guangzhou, Guangdong \\ 510303, P.R. China \\ Full list of author information is \\ available at the end of the article
}

\begin{abstract}
By applying the way of real and functional analysis and estimating the weight functions, we build some lemmas and deduce some Hilbert-type and

Hilbert-Hardy-type integral inequalities with the best possible constant factors. The equivalent forms, the reverses and the operator expressions are all considered. The composition formula of two Hilbert-Hardy-type integral operators and some examples are given.
\end{abstract}

MSC: 26D10; 26D15;40A05; 33B15

Keywords: Hilbert-Hardy-type integral inequality; weight function; equivalent form; reverse; composition of operators

\section{Introduction}

Assuming that $f(x), g(y) \geq 0, f, g \in L^{2}\left(\mathbf{R}_{+}\right)=\left\{f ;\|f\|_{2}=\left(\int_{0}^{\infty}|f(x)|^{2} d x\right)^{\frac{1}{2}}<\infty\right\},\|f\|_{2},\|g\|_{2}>$ 0 , we have the following well-known Hilbert integral inequality and the equivalent form $(c f .[1]):$

$$
\begin{aligned}
& \int_{0}^{\infty} \int_{0}^{\infty} \frac{f(x) g(y)}{x+y} d x d y<\pi\|f\|_{2}\|g\|_{2}, \\
& {\left[\int_{0}^{\infty}\left(\int_{0}^{\infty} \frac{f(x)}{x+y} d x\right)^{2} d y\right]^{\frac{1}{2}}<\pi\|f\|_{2},}
\end{aligned}
$$

where the constant factor $\pi$ is the best possible.

In 1925, by introducing a pair of conjugate exponents $(p, q)\left(\frac{1}{p}+\frac{1}{q}=1\right)$, Hardy [2] gave some extensions of (1) and (2) as follows: For $p>1, f(x), g(y) \geq 0, f \in L^{p}\left(\mathbf{R}_{+}\right), g \in L^{q}\left(\mathbf{R}_{+}\right)$, $\|f\|_{p},\|g\|_{q}>0$, we have the following Hardy-Hilbert integral inequality and the equivalent form:

$$
\begin{aligned}
& \int_{0}^{\infty} \int_{0}^{\infty} \frac{f(x) g(y)}{x+y} d x d y<\frac{\pi}{\sin (\pi / p)}\|f\|_{p}\|g\|_{q}, \\
& {\left[\int_{0}^{\infty}\left(\int_{0}^{\infty} \frac{f(x)}{x+y} d x\right)^{p} d y\right]^{\frac{1}{p}}<\frac{\pi}{\sin (\pi / p)}\|f\|_{p},}
\end{aligned}
$$

where the constant factor $\frac{\pi}{\sin (\pi / p)}$ is the best possible. For $p=q=2$, inequalities (3) and (4) reduce respectively to (1) and (2).

2015 Yang and Chen; licensee Springer. This is an Open Access article distributed under the terms of the Creative Commons Attribution License (http://creativecommons.org/licenses/by/4.0), which permits unrestricted use, distribution, and reproduction in any medium, provided the original work is properly credited. 
Definition 1 If $\lambda \in \mathbf{R}=(-\infty, \infty), \mathbf{R}_{+}=(0, \infty), k_{\lambda}(x, y)$ is a measurable function in $\mathbf{R}_{+}^{2}=$ $\mathbf{R}_{+} \times \mathbf{R}_{+}$, satisfying for any $t, x, y \in \mathbf{R}_{+}, k_{\lambda}(t x, t y)=t^{-\lambda} k_{\lambda}(x, y)$, then we call $k_{\lambda}(x, y)$ homogeneous function of degree $-\lambda$.

In 1934, by using a general non-negative homogeneous function of degree -1 as $k_{1}(x, y)$, Hardy et al. [3] gave some extensions of (3) and (4) as follows: For $p>1, k_{p}=$ $\int_{0}^{\infty} k_{1}(u, 1) u^{\frac{-1}{p}} d u \in \mathbf{R}_{+}, f(x), g(y) \geq 0, f \in L^{p}\left(\mathbf{R}_{+}\right), g \in L^{q}\left(\mathbf{R}_{+}\right),\|f\|_{p},\|g\|_{q}>0$, we have the following Hardy-Hilbert-type integral inequality and the equivalent form:

$$
\begin{aligned}
& \int_{0}^{\infty} \int_{0}^{\infty} k_{1}(x, y) f(x) g(y) d x d y<k_{p}\|f\|_{p}\|g\|_{q}, \\
& {\left[\int_{0}^{\infty}\left(\int_{0}^{\infty} k_{1}(x, y) f(x) d x\right)^{p} d y\right]^{\frac{1}{p}}<k_{p}\|f\|_{p},}
\end{aligned}
$$

where the constant factor $k_{p}$ is the best possible. Some applications of (5) and (6) are provided in [4].

In 1998, by introducing an independent parameter $\lambda \in(0,1]$, Yang [5] gave an extension of (3) with the homogeneous kernel of degree $-\lambda$ as $\frac{1}{(x+y)^{\lambda}}$. In 2009, by using a general nonnegative homogeneous function of degree $-\lambda$ as $k_{\lambda}(x, y)$ and adding another pair of conjugate exponents $(r, s)\left(\frac{1}{r}+\frac{1}{s}=1\right)$, Yang [6] gave some extensions of (5) and (6) as follows: For $p, r>1, \Phi(x)=x^{p\left(1-\frac{\lambda}{r}\right)-1}, \Psi(y)=y^{q\left(1-\frac{\lambda}{s}\right)-1}\left(x, y \in \mathbf{R}_{+}\right), k_{\lambda}(r)=\int_{0}^{\infty} k_{\lambda}(u, 1) u^{\frac{\lambda}{r}-1} d u \in \mathbf{R}_{+}$, $f(x), g(y) \geq 0$,

$$
f \in L_{p, \Phi}\left(\mathbf{R}_{+}\right)=\left\{f ;\|f\|_{p, \Phi}=\left(\int_{0}^{\infty} \Phi(x)|f(x)|^{p} d x\right)^{\frac{1}{p}}<\infty\right\},
$$

$g \in L_{q, \Psi}\left(\mathbf{R}_{+}\right),\|f\|_{p, \Phi},\|g\|_{q, \Psi}>0$, we have the following Yang-Hilbert-type integral inequality and the equivalent form:

$$
\begin{aligned}
& \int_{0}^{\infty} \int_{0}^{\infty} k_{\lambda}(x, y) f(x) g(y) d x d y<k_{\lambda}(r)\|f\|_{p, \Phi}\|g\|_{q, \Psi}, \\
& {\left[\int_{0}^{\infty} y^{\frac{p \lambda}{s}-1}\left(\int_{0}^{\infty} k_{\lambda}(x, y) f(x) d x\right)^{p} d y\right]^{\frac{1}{p}}<k_{\lambda}(r)\|f\|_{p, \Phi},}
\end{aligned}
$$

where the constant factor $k_{\lambda}(r)$ is the best possible.

Remark 1 (i) When $\lambda=1, r=q, s=p$, (7) and (8) reduce respectively to (5) and (6). (ii) By (8), setting $y=\frac{1}{z}$, we have the following Yang-Hilbert-type inequality with the best possible constant factor and a non-homogeneous kernel:

$$
\left[\int_{0}^{\infty} z^{\frac{p \lambda}{r}-1}\left(\int_{0}^{\infty} k_{\lambda}(x z, 1) f(x) d x\right)^{p} d z\right]^{\frac{1}{p}}<k_{\lambda}(r)\|f\|_{p, \Phi} .
$$

Using (2), we may define Hilbert's integral operator $T: L^{2}\left(\mathbf{R}_{+}\right) \rightarrow L^{2}\left(\mathbf{R}_{+}\right)$as follows (cf. [7]): For any $f \in L^{2}\left(\mathbf{R}_{+}\right)$, there exists $T f \in L^{2}\left(\mathbf{R}_{+}\right)$satisfying

$$
T f(y)=\int_{0}^{\infty} \frac{f(x)}{x+y} d x \quad\left(y \in \mathbf{R}_{+}\right) .
$$


Then by (2) we have $\|T f\|_{2} \leq \pi\|f\|_{2}$, and $T$ is a bounded linear operator satisfying $\|T\| \leq \pi$. Since the constant factor in (2) is the best possible, we have $\|T\|=\pi$.

About the discrete forms of (1) and (2), in 1950, Wilhelm [8] gave an operator expression. In 2002, by using the operator theory, Zhang [9] gave some improvements of (2) and the discrete form. In 2006 to 2009, [10] considered a new Hilbert-type operator and its applications, and [11] and [12] gave some multiple Hilbert-type operator expressions.

By using (8), we can define the Yang-Hilbert-type integral operator $T: L_{p, \Phi}\left(\mathbf{R}_{+}\right) \rightarrow$ $L_{p, \Phi}\left(\mathbf{R}_{+}\right)$as follows (cf. [6]): For any $f \in L_{p, \Phi}\left(\mathbf{R}_{+}\right)$, there exists $T f \in L_{p, \Phi}\left(\mathbf{R}_{+}\right)$satisfying

$$
T f(y)=y^{\lambda-1} \int_{0}^{\infty} \frac{f(x)}{x+y} d x \quad\left(y \in \mathbf{R}_{+}\right) .
$$

Then by (8) we have $\|T f\|_{p, \Phi} \leq k_{\lambda}(r)\|f\|_{p, \Phi}$, and $T$ is a bounded linear operator satisfying $\|T\| \leq k_{\lambda}(r)$. Since the constant factor in (8) is the best possible, we have $\|T\|=k_{\lambda}(r)$.

About the composition of two Hilbert-type operators, the main objective is to build the expression $\left\|T_{1} T_{2}\right\|=\left\|T_{1}\right\| \cdot\left\|T_{2}\right\|$. Recently, [13] published a composition of two discrete Hilbert-Hardy-type operators with particular kernels. Adiyasuren et al. [14] published a composition of two half-discrete Hilbert-Hardy-type operators with some particular kernels, and [15] and [16] published some composition of two Hilbert-Hardy-type integral operators with particular kernels. These works are hard and interesting.

In this paper, applying the way of real and functional analysis and estimating the weight functions, we build some lemmas and deduce some Hilbert-type and Hilbert-Hardy-type integral inequalities with the best possible constant factors. The equivalent forms, the reverses and the operator expressions are all considered. The composition formulas of two Hilbert-Hardy-type integral operators and some examples are given, which are some extensions of the results of [15] and [16].

\section{Some lemmas}

In the following, we agree on that $p>0(p \neq 1), \frac{1}{p}+\frac{1}{q}=1$.

Lemma 1 (cf. [17], Lemma 2.2.5) Suppose that $\lambda \in A=(0, c)(0<c \leq \infty), k_{\lambda}^{(s)}(x, y)$ are nonnegative homogeneous functions of degree $-\lambda$ in $\mathbf{R}_{+}^{2}$,

$$
k^{(s)}\left(\frac{\lambda}{2}\right):=\int_{0}^{\infty} k_{\lambda}^{(s)}(u, 1) u^{\frac{\lambda}{2}-1} d u \quad(s=1,2,3)
$$

there exists a constant $\delta_{0} \in\left(0, \frac{\lambda}{2}\right)$ such that $k^{(s)}\left(\frac{\lambda}{2} \pm \delta_{0}\right) \in \mathbf{R}_{+}$. Then, for any $\delta \in\left[0, \delta_{0}\right)$, we have $k^{(s)}\left(\frac{\lambda}{2} \pm \delta\right) \in \mathbf{R}_{+}$and

$$
\lim _{\delta \rightarrow 0^{+}} k^{(s)}\left(\frac{\lambda}{2} \pm \delta\right)=k^{(s)}\left(\frac{\lambda}{2}\right) \quad(s=1,2,3) .
$$

With the assumptions of Lemma 1, we set the following conditions.

Condition (i) For $\lambda \in A$, there exist constants $\delta_{1} \in\left(0, \delta_{0}\right)$ and $L_{1}>0$ such that

$$
k_{\lambda}^{(s)}(u, 1) u^{\frac{\lambda}{2}+\delta_{1}} \leq L_{1} \quad(u \in(1, \infty) ; s=2,3) .
$$


Condition (ii) For $\lambda \in(0,1) \cap A$, there exists a constant $L_{2}>0$ such that

$$
k_{\lambda}^{(s)}(u, 1)(u-1)^{\lambda} \leq L_{2} \quad(u \in(1, \infty) ; s=2,3) .
$$

Condition (iii) For $\lambda \in(0,1) \cap A$, there exist constants $a \in(0, \lambda)$ and $L_{3}>0$ such that

$$
k_{\lambda}^{(1)}(u, 1) u^{a} \leq L_{3} \quad(u \in(0, \infty)) .
$$

Example 1 For $\lambda \in A=(0, \infty), s=1,2,3$, the functions

$$
k_{\lambda}^{(s)}(u, 1)=\frac{1}{(u+1)^{\lambda}}, \frac{1}{u^{\lambda}+1}, \frac{\ln u}{u^{\lambda}-1}, \frac{|\ln u|^{\beta-1}}{(\max \{u, 1\})^{\lambda}} \quad(\beta \geq 1)
$$

satisfy Conditions (i) and (iii). In fact, for $b=\frac{\lambda}{2}+\delta_{1}$ or $b=a \in(0, \lambda)$, we find

$$
\lim _{u \rightarrow 0^{+}} k_{\lambda}^{(s)}(u, 1) u^{b}=\lim _{u \rightarrow \infty} k_{\lambda}^{(s)}(u, 1) u^{b}=0 .
$$

In view of the continuity, $k_{\lambda}^{(s)}(u, 1) u^{b}(s=1,2,3)$ are bounded in $(0, \infty)$ and then satisfy (11) and (13).

It is evident that for $\lambda \in A=(0,1)$, the functions

$$
k_{\lambda}^{(s)}(u, 1)=\frac{1}{(u-1)^{\lambda}} \quad(u \in(1, \infty) ; s=2,3)
$$

satisfy Condition (ii).

Definition 2 With the assumptions of Lemma 1 and Condition (i), we define the following two sequences of real functions:

$$
\begin{aligned}
& \widetilde{F}_{k}(y):= \begin{cases}y^{\lambda-1} \int_{0}^{1} k_{\lambda}^{(2)}(x, y) x^{\frac{\lambda}{2}+\frac{1}{p k}-1} d x, & y \in(0,1), \\
0, & y \in[1, \infty),\end{cases} \\
& \widetilde{G}_{k}(x):= \begin{cases}x^{\lambda-1} \int_{1}^{\infty} k_{\lambda}^{(3)}(x, y) y^{\frac{\lambda}{2}-\frac{1}{q k}-1} d y, & x \in(1, \infty), \\
0, & x \in(0,1],\end{cases}
\end{aligned}
$$

where $k>\max \left\{\frac{1}{|q| \delta_{1}}, \frac{1}{p \delta_{1}}\right\}(k \in \mathbf{N}=\{1,2, \ldots\})$.

Setting $u=x / y(0<y<1)$, we find

$$
\begin{aligned}
\widetilde{F}_{k}(y) & =y^{\frac{\lambda}{2}+\frac{1}{p k}-1} \int_{0}^{\frac{1}{y}} k_{\lambda}^{(2)}(u, 1) u^{\frac{\lambda}{2}+\frac{1}{p k}-1} d u \\
& =y^{\frac{\lambda}{2}+\frac{1}{p k}-1}\left(\int_{0}^{\infty} k_{\lambda}^{(2)}(u, 1) u^{\frac{\lambda}{2}+\frac{1}{p k}-1} d u-\int_{\frac{1}{y}}^{\infty} k_{\lambda}^{(2)}(u, 1) u^{\frac{\lambda}{2}+\frac{1}{p k}-1} d u\right) \\
& =y^{\frac{\lambda}{2}+\frac{1}{p k}-1} k^{(2)}\left(\frac{\lambda}{2}+\frac{1}{p k}\right)-F(y), \\
F(y) & :=y^{\frac{\lambda}{2}+\frac{1}{p k}-1} \int_{\frac{1}{y}}^{\infty} k_{\lambda}^{(2)}(u, 1) u^{\frac{\lambda}{2}+\frac{1}{p k}-1} d u \quad(y \in(0,1)) .
\end{aligned}
$$


(a) If $k_{\lambda}^{(2)}(u, 1)$ satisfies Condition (i) (for $\lambda \in A$ ), then by (11) we have

$$
0 \leq F(y) \leq L_{1} y^{\frac{\lambda}{2}+\frac{1}{p k}-1} \int_{\frac{1}{y}}^{\infty} u^{-\frac{\lambda}{2}-\delta_{1}} u^{\frac{\lambda}{2}+\frac{1}{p k}-1} d u=\frac{L_{1} y^{\frac{\lambda}{2}+\delta_{1}-1}}{\delta_{1}-\frac{1}{p k}} \quad(y \in(0,1)) .
$$

(b) If $k_{\lambda}^{(2)}(u, 1)$ satisfies Condition (ii) (for $\lambda \in(0,1) \cap A$ ), then by (12) we have

$$
\begin{aligned}
0 & \leq F(y) \leq L_{2} y^{\frac{\lambda}{2}+\frac{1}{p k}-1} \int_{\frac{1}{y}}^{\infty} \frac{u^{\frac{\lambda}{2}+\frac{1}{p k}-1}}{(u-1)^{\lambda}} d u \stackrel{v=1 /(y u)}{=} L_{2} y^{\lambda-1} \int_{0}^{1} \frac{v^{\frac{\lambda}{2}-\frac{1}{p k}-1}}{(1-y v)^{\lambda}} d v \\
& \leq \frac{L_{2} y^{\lambda-1}}{(1-y)^{\lambda}} \int_{0}^{1} v^{\frac{\lambda}{2}-\frac{1}{p k}-1} d v=\frac{L_{2}}{\frac{\lambda}{2}-\frac{1}{p k}} \frac{y^{\lambda-1}}{(1-y)^{\lambda}} \quad(y \in(0,1)) .
\end{aligned}
$$

Still setting $u=x / y(x>1)$, we obtain

$$
\begin{aligned}
\widetilde{G}_{k}(x) & =x^{\frac{\lambda}{2}-\frac{1}{q k}-1} \int_{0}^{x} k_{\lambda}^{(3)}(u, 1) u^{\frac{\lambda}{2}+\frac{1}{q k}-1} d u \\
& =x^{\frac{\lambda}{2}-\frac{1}{q k}-1}\left(\int_{0}^{\infty} k_{\lambda}^{(3)}(u, 1) u^{\frac{\lambda}{2}+\frac{1}{q k}-1} d u-\int_{x}^{\infty} k_{\lambda}^{(3)}(u, 1) u^{\frac{\lambda}{2}+\frac{1}{q k}-1} d u\right) \\
& =x^{\frac{\lambda}{2}-\frac{1}{q k}-1} k^{(3)}\left(\frac{\lambda}{2}+\frac{1}{q k}\right)-G(x), \\
G(x) & :=x^{\frac{\lambda}{2}-\frac{1}{q k}-1} \int_{x}^{\infty} k_{\lambda}^{(3)}(u, 1) u^{\frac{\lambda}{2}+\frac{1}{q k}-1} d u \quad(x \in(1, \infty)) .
\end{aligned}
$$

(c) If $k_{\lambda}^{(3)}(u, 1)$ satisfies Condition (i) (for $\lambda \in A$ ), then by (11) we have

$$
0 \leq G(x) \leq L_{1} x^{\frac{\lambda}{2}-\frac{1}{q k}-1} \int_{x}^{\infty} u^{-\frac{\lambda}{2}-\delta_{1}} u^{\frac{\lambda}{2}+\frac{1}{q k}-1} d u=\frac{L_{1} x^{\frac{\lambda}{2}-\delta_{1}-1}}{\delta_{1}-\frac{1}{q k}} \quad(x \in(1, \infty)) .
$$

(d) If $k_{\lambda}^{(3)}(u, 1)$ satisfies Condition (ii) (for $\lambda \in(0,1) \cap A$ ), then by (12) we have

$$
\begin{aligned}
0 & \leq G(x) \leq L_{2} x^{\frac{\lambda}{2}-\frac{1}{q k}-1} \int_{x}^{\infty} \frac{u^{\frac{\lambda}{2}+\frac{1}{q k}-1}}{(u-1)^{\lambda}} d u \stackrel{v=x / u}{=} L_{2} x^{\lambda-1} \int_{0}^{1} \frac{v^{\frac{\lambda}{2}-\frac{1}{q k}-1}}{(x-v)^{\lambda}} d v \\
& \leq \frac{L_{2} x^{\lambda-1}}{(x-1)^{\lambda}} \int_{0}^{1} v^{\frac{\lambda}{2}-\frac{1}{q k}-1} d v=\frac{L_{2} x^{\lambda-1}}{\left(\frac{\lambda}{2}-\frac{1}{q k}\right)(x-1)^{\lambda}} \quad(x \in(1, \infty)) .
\end{aligned}
$$

Remark 2 In view of the results of (a)-(d), there exists a large constant $L>0$ such that

(a) $F(y) \leq L y^{\frac{\lambda}{2}+\delta_{1}-1}(y \in(0,1) ; \lambda \in A)$;

(b) $F(y) \leq L \frac{y^{\lambda-1}}{(1-y)^{\lambda}}(y \in(0,1) ; \lambda \in(0,1) \cap A)$;

(c) $G(x) \leq L x^{\frac{\lambda}{2}-\delta_{1}-1}(x \in(1, \infty) ; \lambda \in A)$;

(d) $G(x) \leq L \frac{x^{\lambda-1}}{(x-1)^{\lambda}}(x \in(1, \infty) ; \lambda \in(0,1) \cap A)$.

Lemma 2 With the assumptions of Lemma $1,(1) k_{\lambda}^{(2)}(u, 1)\left(k_{\lambda}^{(3)}(u, 1)\right)$ satisfies Condition (i) or Condition (ii); (2) if $k_{\lambda}^{(s)}(u, 1)(s=2,3)$ only satisfy Condition (i), then $\lambda \in A$; otherwise, $\lambda \in(0,1) \cap A$. Then we have

$$
\widetilde{L}_{k}:=\frac{1}{k} \int_{0}^{\infty} \int_{0}^{\infty} k_{\lambda}^{(1)}(x y, 1) \widetilde{F}_{k}(y) \widetilde{G}_{k}(x) d y d x \geq \prod_{i=1}^{3} k_{\lambda}^{(i)}\left(\frac{\lambda}{2}\right)+o(1) \quad(k \rightarrow \infty) .
$$


Yang and Chen Journal of Inequalities and Applications (2015) 2015:100

Page 6 of 18

Proof In view of (15) and (16), we have

$$
\begin{aligned}
\widetilde{L}_{k}= & \frac{1}{k} \int_{1}^{\infty} \int_{0}^{1} k_{\lambda}^{(1)}(x y, 1)\left[y^{\frac{\lambda}{2}+\frac{1}{p k}-1} k_{\lambda}^{(2)}\left(\frac{\lambda}{2}+\frac{1}{p k}\right)-F(y)\right] \\
& \times\left[x^{\frac{\lambda}{2}-\frac{1}{q k}-1} k_{\lambda}^{(3)}\left(\frac{\lambda}{2}+\frac{1}{q k}\right)-G(x)\right] d y d x=I_{1}-I_{2}-I_{3}+I_{4},
\end{aligned}
$$

where we define

$$
\begin{aligned}
I_{1}:= & \frac{1}{k} k_{\lambda}^{(2)}\left(\frac{\lambda}{2}+\frac{1}{p k}\right) k_{\lambda}^{(3)}\left(\frac{\lambda}{2}+\frac{1}{q k}\right) \\
& \times \int_{1}^{\infty}\left(\int_{0}^{1} k_{\lambda}^{(1)}(x y, 1) y^{\frac{\lambda}{2}+\frac{1}{p k}-1} d y\right) x^{\frac{\lambda}{2}-\frac{1}{q k}-1} d x, \\
I_{2}:= & \frac{1}{k} k_{\lambda}^{(3)}\left(\frac{\lambda}{2}+\frac{1}{q k}\right) \int_{1}^{\infty}\left(\int_{0}^{1} k_{\lambda}^{(1)}(x y, 1) F(y) d y\right) x^{\frac{\lambda}{2}-\frac{1}{q k}-1} d x, \\
I_{3}:= & \frac{1}{k} k_{\lambda}^{(2)}\left(\frac{\lambda}{2}+\frac{1}{p k}\right) \int_{1}^{\infty}\left(\int_{0}^{1} k_{\lambda}^{(1)}(x y, 1) y^{\frac{\lambda}{2}+\frac{1}{p k}-1} d y\right) G(x) d x, \\
I_{4}:= & \frac{1}{k} \int_{1}^{\infty}\left(\int_{0}^{1} k_{\lambda}^{(1)}(x y, 1) F(y) d y\right) G(x) d x .
\end{aligned}
$$

It is evident that

$$
I_{1}-I_{2}-I_{3} \leq \widetilde{L}_{k} \leq I_{1}+I_{4}
$$

By Fubini's theorem, we obtain that (cf. [18])

$$
\begin{aligned}
\int_{1}^{\infty} & \left(\int_{0}^{1} k_{\lambda}^{(1)}(x y, 1) y^{\frac{\lambda}{2}+\frac{1}{p k}-1} d y\right) x^{\frac{\lambda}{2}-\frac{1}{q k}-1} d x \\
\stackrel{u=x y}{=} & \int_{1}^{\infty}\left(\int_{0}^{x} k_{\lambda}^{(1)}(u, 1) u^{\frac{\lambda}{2}+\frac{1}{p k}-1} d u\right) x^{-\frac{1}{k}-1} d x \\
= & \int_{1}^{\infty}\left(\int_{0}^{1} k_{\lambda}^{(1)}(u, 1) u^{\frac{\lambda}{2}+\frac{1}{p k}-1} d u\right) x^{-\frac{1}{k}-1} d x \\
& +\int_{1}^{\infty}\left(\int_{1}^{x} k_{\lambda}^{(1)}(u, 1) u^{\frac{\lambda}{2}+\frac{1}{p k}-1} d u\right) x^{-\frac{1}{k}-1} d x \\
= & k \int_{0}^{1} k_{\lambda}^{(1)}(u, 1) u^{\frac{\lambda}{2}+\frac{1}{p k}-1} d u \\
& +\int_{1}^{\infty}\left(\int_{u}^{\infty} x^{-\frac{1}{k}-1} d x\right) k_{\lambda}^{(1)}(u, 1) u^{\frac{\lambda}{2}+\frac{1}{p k}-1} d u \\
= & k\left(\int_{0}^{1} k_{\lambda}^{(1)}(u, 1) u^{\frac{\lambda}{2}+\frac{1}{p k}-1} d u+\int_{1}^{\infty} k_{\lambda}^{(1)}(u, 1) u^{\frac{\lambda}{2}-\frac{1}{q k}-1} d u\right) .
\end{aligned}
$$

Since $\left\{k_{\lambda}^{(1)}(u, 1) u^{\frac{\lambda}{2}+\frac{1}{p k}-1}\right\}_{k=1}^{\infty}(u \in(0,1))$ is increasing, by Levi's theorem (cf. [18]), it follows that

$$
\int_{0}^{1} k_{\lambda}^{(1)}(u, 1) u^{\frac{\lambda}{2}+\frac{1}{p k}-1} d u \rightarrow \int_{0}^{1} k_{\lambda}^{(1)}(u, 1) u^{\frac{\lambda}{2}-1} d u \quad(k \rightarrow \infty)
$$


Since $k_{\lambda}^{(1)}(u, 1) u^{\frac{\lambda}{2}-\frac{1}{q k}-1} \leq k_{\lambda}^{(1)}(u, 1) u^{\mu+\delta_{1}-1}(u \in(1, \infty))$ and

$$
0 \leq \int_{1}^{\infty} k_{\lambda}^{(1)}(u, 1) u^{\frac{\lambda}{2}+\delta_{1}-1} d u \leq k^{(1)}\left(\frac{\lambda}{2}+\delta_{1}\right)<\infty
$$

then by the Lebesgue convergence control theorem $(c f .[18])$, we have

$$
\int_{1}^{\infty} k_{\lambda}^{(1)}(u, 1) u^{\frac{\lambda}{2}-\frac{1}{q k}-1} d u \rightarrow \int_{1}^{\infty} k_{\lambda}^{(1)}(u, 1) u^{\frac{\lambda}{2}-1} d u \quad(k \rightarrow \infty)
$$

Hence, by Lemma 1, we find, for $k \rightarrow \infty$,

$$
\begin{aligned}
I_{1}= & k^{(2)}\left(\frac{\lambda}{2}+\frac{1}{p k}\right) k^{(3)}\left(\frac{\lambda}{2}+\frac{1}{q k}\right) \\
& \times\left(\int_{0}^{1} k_{\lambda}^{(1)}(u, 1) u^{\frac{\lambda}{2}+\frac{1}{p k}-1} d u+\int_{1}^{\infty} k_{\lambda}^{(1)}(u, 1) u^{\frac{\lambda}{2}-\frac{1}{q k}-1} d u\right) \rightarrow \prod_{s=1}^{3} k^{(s)}\left(\frac{\lambda}{2}\right) .
\end{aligned}
$$

(1) We estimate $I_{2}$.

(a) If $k_{\lambda}^{(2)}(u, 1)$ satisfies Condition (i) for $\lambda \in A$, then by Remark 2(a) we have

$$
\begin{array}{rl}
0 & \leq J_{2}:=\int_{1}^{\infty}\left(\int_{0}^{1} k_{\lambda}^{(1)}(x y, 1) F(y) d y\right) x^{\frac{\lambda}{2}-\frac{1}{q k}-1} d x \\
& \leq L \int_{1}^{\infty}\left(\int_{0}^{\infty} k_{\lambda}^{(1)}(x y, 1) y^{\frac{\lambda}{2}+\delta_{1}-1} d y\right) x^{\frac{\lambda}{2}-\frac{1}{q k}-1} d x \\
\stackrel{u}{u}=x y & L \int_{1}^{\infty}\left(\int_{0}^{\infty} k_{\lambda}^{(1)}(u, 1) u^{\frac{\lambda}{2}+\delta_{1}-1} d u\right) x^{-\delta_{1}-\frac{1}{q k}-1} d x \\
& =\frac{L \cdot k_{\lambda}^{(1)}\left(\frac{\lambda}{2}+\delta_{1}\right)}{\delta_{1}+\frac{1}{q k}}<\infty .
\end{array}
$$

(b) If $k_{\lambda}^{(2)}(u, 1)$ satisfies Condition (ii) for $\lambda \in(0,1) \cap A$, then by Remark 2(b) we have

$$
\begin{aligned}
0 & \leq J_{2} \leq L \int_{0}^{1}\left(\int_{0}^{\infty} k_{\lambda}^{(1)}(x y, 1) x^{\frac{\lambda}{2}-\frac{1}{q k}-1} d x\right) \frac{y^{\lambda-1}}{(1-y)^{\lambda}} d y \\
& \stackrel{u=x y}{=} L \int_{0}^{1}\left(\int_{0}^{\infty} k_{\lambda}^{(1)}(u, 1) u^{\frac{\lambda}{2}-\frac{1}{q k}-1} d u\right) \frac{y^{\frac{\lambda}{2}+\frac{1}{q k}-1}}{(1-y)^{\lambda}} d y \\
& =L \cdot k^{(1)}\left(\frac{\lambda}{2}-\frac{1}{q k}\right) B\left(1-\lambda, \frac{\lambda}{2}+\frac{1}{q k}\right)<\infty .
\end{aligned}
$$

Therefore, in view of (a) and (b), we have $I_{2} \rightarrow 0(k \rightarrow \infty)$.

(2) We estimate $I_{3}$.

(c) If $k_{\lambda}^{(3)}(u, 1)$ satisfies Condition (i) for $\lambda \in A$, then by Remark 2(c) we have

$$
\begin{aligned}
0 & \leq J_{3}:=\int_{1}^{\infty}\left(\int_{0}^{1} k_{\lambda}^{(1)}(x y, 1) y^{\frac{\lambda}{2}+\frac{1}{p k}-1} d y\right) G(x) d x \\
& \leq L \int_{1}^{\infty}\left(\int_{0}^{\infty} k_{\lambda}^{(1)}(x y, 1) y^{\frac{\lambda}{2}+\frac{1}{p k}-1} d y\right) x^{\frac{\lambda}{2}-\delta_{1}-1} d x
\end{aligned}
$$




$$
\begin{aligned}
& \stackrel{u=x y}{=} L \int_{1}^{\infty}\left(\int_{0}^{\infty} k_{\lambda}^{(1)}(u, 1) u^{\frac{\lambda}{2}+\frac{1}{p k}-1} d u\right) x^{-\delta_{1}-\frac{1}{p k}-1} d x \\
& =\frac{L \cdot k^{(1)}\left(\frac{\lambda}{2}+\frac{1}{p k}\right)}{\delta_{1}+\frac{1}{p k}}<\infty .
\end{aligned}
$$

(d) If $k_{\lambda}^{(3)}(u, 1)$ satisfies Condition (ii) for $\lambda \in(0,1) \cap A$, then by Remark 2(d), we have

$$
\begin{aligned}
& 0 \leq J_{3} \leq L \int_{1}^{\infty}\left(\int_{0}^{\infty} k_{\lambda}^{(1)}(x y, 1) y^{\frac{\lambda}{2}+\frac{1}{p k}-1} d y\right) \frac{x^{\lambda-1}}{(x-1)^{\lambda}} d x \\
& \stackrel{u=x y}{=} L \int_{1}^{\infty}\left(\int_{0}^{\infty} k_{\lambda}^{(1)}(u, 1) u^{\frac{\lambda}{2}+\frac{1}{p k}-1} d u\right) \frac{x^{\frac{\lambda}{2}-\frac{1}{p k}-1}}{(x-1)^{\lambda}} d x \\
&=L \cdot k_{\lambda}^{(1)}\left(\frac{\lambda}{2}+\frac{1}{p k}\right) B\left(1-\lambda, \frac{\lambda}{2}+\frac{1}{p k}\right)<\infty .
\end{aligned}
$$

Therefore, in view of (c) and (d), we have $I_{3} \rightarrow 0(k \rightarrow \infty)$.

By (19) and the above results, we have (17).

Lemma 3 Suppose that $(1) \lambda \in A=(0, c)(0<c \leq \infty), k_{\lambda}^{(s)}(x, y)$ are non-negative homogeneous functions of degree $-\lambda$ in $\mathbf{R}_{+}^{2}$,

$$
k^{(s)}\left(\frac{\lambda}{2}\right)=\int_{0}^{\infty} k_{\lambda}^{(s)}(u, 1) u^{\frac{\lambda}{2}-1} d u \quad(s=1,2,3)
$$

there exists a constant $\delta_{0} \in\left(0, \frac{\lambda}{2}\right)$ such that $k^{(s)}\left(\frac{\lambda}{2} \pm \delta_{0}\right) \in \mathbf{R}_{+} ;(2) k_{\lambda}^{(2)}(u, 1)\left(k_{\lambda}^{(3)}(u, 1)\right)$ satisfies Condition (i) or Condition (ii); (3) if both $k_{\lambda}^{(2)}(u, 1)$ and $k_{\lambda}^{(3)}(u, 1)$ satisfy Condition (ii), then $k_{\lambda}^{(1)}(u, 1)$ satisfies Condition (iii); (4) if $k_{\lambda}^{(s)}(u, 1)(s=2,3)$ only satisfy Condition (i), then $\lambda \in$ $A$; otherwise, $\lambda \in(0,1) \cap A$. Then we have the reverse of (17), namely

$$
\begin{aligned}
\widetilde{L}_{k} & =\frac{1}{k} \int_{0}^{\infty} \int_{0}^{\infty} k_{\lambda}^{(1)}(x y, 1) \widetilde{F}_{k}(y) \widetilde{G}_{k}(x) d y d x \\
& =\prod_{s=1}^{3} k^{(s)}\left(\frac{\lambda}{2}\right)+o(1) \quad(k \rightarrow \infty) .
\end{aligned}
$$

Proof We have four cases to show that in any case, $I_{4} \rightarrow 0(k \rightarrow \infty)$.

Case (i). $\lambda \in A, F(y) \leq L y^{\frac{\lambda}{2}+\delta_{1}-1}(y \in(0,1)), G(x) \leq L x^{\frac{\lambda}{2}-\delta_{1}-1}(x \in(1, \infty))$. We have

$$
\begin{aligned}
& J_{4}:=\int_{1}^{\infty}\left(\int_{0}^{1} k_{\lambda}^{(1)}(x y, 1) F(y) d y\right) G(x) d x \\
& \leq L^{2} \int_{1}^{\infty}\left(\int_{0}^{\infty} k_{\lambda}^{(1)}(x y, 1) y^{\frac{\lambda}{2}+\delta_{1}-1} d y\right) x^{x^{\frac{\lambda}{2}-\delta_{1}-1}} d x \\
& \stackrel{u=x y}{=} L^{2} \int_{1}^{\infty}\left(\int_{0}^{\infty} k_{\lambda}^{(1)}(u, 1) u^{\frac{\lambda}{2}+\delta_{1}-1} d u\right) x^{-2 \delta_{1}-1} d x \\
& =\frac{L^{2}}{2 \delta_{1}} k^{(1)}\left(\frac{\lambda}{2}+\delta_{1}\right)<\infty \text {. }
\end{aligned}
$$


Case (ii). $\lambda \in(0,1) \cap A, F(y) \leq L y^{\frac{\lambda}{2}+\delta_{1}-1}(y \in(0,1)), G(x) \leq L \frac{x^{\lambda-1}}{(x-1)^{\lambda}}(x \in(1, \infty))$. We have

$$
\begin{aligned}
J_{4} & \leq L^{2} \int_{1}^{\infty}\left(\int_{0}^{\infty} k_{\lambda}^{(1)}(x y, 1) y^{\frac{\lambda}{2}+\delta_{1}-1} d y\right) \frac{x^{\lambda-1}}{(x-1)^{\lambda}} d x \\
& \stackrel{u=x y}{=} L^{2} \int_{1}^{\infty}\left(\int_{0}^{\infty} k_{\lambda}^{(1)}(u, 1) u^{\frac{\lambda}{2}+\delta_{1}-1} d u\right) \frac{x^{\frac{\lambda}{2}-\delta_{1}-1}}{(x-1)^{\lambda}} d x \\
& =L^{2} k^{(1)}\left(\frac{\lambda}{2}+\delta_{1}\right) B\left(1-\lambda, \frac{\lambda}{2}+\delta_{1}\right)<\infty .
\end{aligned}
$$

Case (iii). $\lambda \in(0,1) \cap A, F(y) \leq L \frac{y^{\lambda-1}}{(1-y)^{\lambda}}(y \in(0,1)), G(x) \leq L x^{\frac{\lambda}{2}-\delta_{1}-1}(x \in(1, \infty))$. We have

$$
\begin{aligned}
J_{4} & \leq L^{2} \int_{0}^{1}\left(\int_{0}^{\infty} k_{\lambda}^{(1)}(x y, 1) x^{\frac{\lambda}{2}-\delta_{1}-1} d x\right) \frac{y^{\lambda-1}}{(1-y)^{\lambda}} d y \\
& \stackrel{u=x y}{=} L^{2} \int_{0}^{1}\left(\int_{0}^{\infty} k_{\lambda}^{(1)}(u, 1) u^{\frac{\lambda}{2}-\delta_{1}-1} d u\right) \frac{y^{\frac{\lambda}{2}+\delta_{1}-1}}{(1-y)^{\lambda}} d y \\
& =L^{2} k^{(1)}\left(\frac{\lambda}{2}-\delta_{1}\right) B\left(1-\lambda, \frac{\lambda}{2}+\delta_{1}\right)<\infty
\end{aligned}
$$

Case (iv). $\lambda \in(0,1) \cap A, F_{k}(y) \leq L \frac{y^{\lambda-1}}{(1-y)^{\lambda}}(y \in(0,1)), G_{k}(x) \leq L \frac{x^{\lambda-1}}{(x-1)^{\lambda}}(x \in(1, \infty)), k_{\lambda}^{(1)}(u, 1)$ satisfies Condition (iii). We have

$$
\begin{aligned}
J_{4} & \leq L^{2} L_{3} \int_{1}^{\infty}\left(\int_{0}^{1}(x y)^{-a} \frac{y^{\lambda-1}}{(1-y)^{\lambda}} d y\right) \frac{x^{\lambda-1}}{(x-1)^{\lambda}} d x \\
& =L^{2} L_{3} \int_{1}^{\infty}\left(\int_{0}^{1} \frac{y^{\lambda-a-1}}{(1-y)^{\lambda}} d y\right) \frac{x^{\lambda-a-1}}{(x-1)^{\lambda}} d x \\
& =L^{2} L_{3} B(1-\lambda, \lambda-a) B(1-\lambda, a)<\infty .
\end{aligned}
$$

Hence, in any case, $I_{4}=\frac{1}{k} J_{4} \rightarrow 0(k \rightarrow \infty)$.

Therefore, by (19) and (20), we have the reverse of (17), and then (21) follows.

\section{Some equivalent Hilbert-type inequalities}

We set functions $\varphi(x):=x^{p\left(1-\frac{\lambda}{2}\right)-1}, \psi(y):=y^{q\left(1-\frac{\lambda}{2}\right)-1}\left(x, y \in \mathbf{R}_{+}\right)$in the following theorem.

Theorem 1 Suppose that $(1) \lambda \in A=(0, c)(0<c \leq \infty), k_{\lambda}^{(s)}(x, y)$ are non-negative homogeneous functions of degree $-\lambda$ in $\mathbf{R}_{+}^{2}$,

$$
k^{(s)}\left(\frac{\lambda}{2}\right)=\int_{0}^{\infty} k_{\lambda}^{(s)}(u, 1) u^{\frac{\lambda}{2}-1} d u \quad(s=1,2,3)
$$

there exists a constant $\delta_{0} \in\left(0, \frac{\lambda}{2}\right)$ such that $k^{(s)}\left(\frac{\lambda}{2} \pm \delta_{0}\right) \in \mathbf{R}_{+} ;(2) k_{\lambda}^{(2)}(u, 1)\left(k_{\lambda}^{(3)}(u, 1)\right)$ satisfies Condition (i) or Condition (ii); (3) if both $k_{\lambda}^{(2)}(u, 1)$ and $k_{\lambda}^{(3)}(u, 1)$ satisfy Condition (ii), then $k_{\lambda}^{(1)}(u, 1)$ satisfies Condition (iii); (4) if $k_{\lambda}^{(s)}(u, 1)(s=2,3)$ only satisfy Condition (i), then $\lambda \in A$; otherwise, $\lambda \in(0,1) \cap A$. For $p>1, f(x), G(y) \geq 0, f \in L_{p, \varphi}\left(\mathbf{R}_{+}\right), G \in L_{q, \psi}\left(\mathbf{R}_{+}\right)$, $\|f\|_{p, \varphi},\|G\|_{q, \psi}>0$, and

$$
F_{\lambda}(y):= \begin{cases}y^{\lambda-1} \int_{0}^{\infty} k_{\lambda}^{(2)}(x, y) f(x) d x, & y \in\{y>0 ; f(y)>0\} \\ 0, & y \in\{y>0 ; f(y)=0\}\end{cases}
$$


we have the following equivalent inequalities:

$$
\begin{aligned}
& I:=\int_{0}^{\infty} \int_{0}^{\infty} k_{\lambda}^{(1)}(x y, 1) F_{\lambda}(y) G(x) d y d x<\prod_{s=1}^{2} k^{(s)}\left(\frac{\lambda}{2}\right)\|f\|_{p, \varphi}\|G\|_{q, \psi}, \\
& J:=\left[\int_{0}^{\infty} x^{\frac{p \lambda}{2}-1}\left(\int_{0}^{\infty} k_{\lambda}^{(1)}(x y, 1) F_{\lambda}(y) d y\right)^{p} d x\right]^{\frac{1}{p}}<\prod_{s=1}^{2} k^{(s)}\left(\frac{\lambda}{2}\right)\|f\|_{p, \varphi},
\end{aligned}
$$

where the constant factor $\prod_{s=1}^{2} k^{(s)}\left(\frac{\lambda}{2}\right)$ is the best possible.

In particular, for $g(y) \geq 0, g \in L_{q, \psi}\left(\mathbf{R}_{+}\right),\|g\|_{q, \psi}>0$, and

$$
G(x)=G_{\lambda}(x):= \begin{cases}x^{\lambda-1} \int_{0}^{\infty} k_{\lambda}^{(3)}(x, y) g(y) d y, & x \in\{x>0 ; g(x)>0\} \\ 0, & x \in\{x>0 ; g(x)=0\}\end{cases}
$$

we have the following inequality:

$$
\int_{0}^{\infty} \int_{0}^{\infty} k_{\lambda}^{(1)}(x y, 1) F_{\lambda}(y) G_{\lambda}(x) d y d x<\prod_{s=1}^{3} k^{(s)}\left(\frac{\lambda}{2}\right)\|f\|_{p, \varphi}\|g\|_{q, \psi},
$$

where the constant factor $\prod_{s=1}^{3} k^{(s)}\left(\frac{\lambda}{2}\right)$ is still the best possible.

Proof By (9) and (8) (for $r=s=2$ ), we have

$$
\begin{aligned}
J=\left[\int_{0}^{\infty} y^{\frac{p \lambda}{2}-1}\left(\int_{0}^{\infty} k_{\lambda}^{(1)}(x y, 1) F_{\lambda}(x) d x\right)^{p} d y\right]^{\frac{1}{p}} \leq k^{(1)}\left(\frac{\lambda}{2}\right)\left\|F_{\lambda}\right\|_{p, \varphi}, \\
\left\|F_{\lambda}\right\|_{p, \varphi}=\left[\int_{0}^{\infty} y^{p\left(1-\frac{\lambda}{2}\right)-1}\left(y^{\lambda-1} \int_{0}^{\infty} k_{\lambda}^{(2)}(x, y) f(x) d x\right)^{p} d y\right]^{\frac{1}{p}} \\
=\left[\int_{0}^{\infty} y^{\frac{p \lambda}{2}-1}\left(\int_{0}^{\infty} k_{\lambda}^{(2)}(x, y) f(x) d x\right)^{p} d y\right]^{\frac{1}{p}} \\
<k^{(2)}\left(\frac{\lambda}{2}\right)\|f\|_{p, \varphi} .
\end{aligned}
$$

Then we have (24).

By Hölder's inequality (cf. [19]), we have

$$
I=\int_{0}^{\infty}\left(x^{\frac{\lambda}{2}-\frac{1}{p}} \int_{0}^{\infty} k_{\lambda}^{(1)}(x y, 1) F_{\lambda}(y) d y\right)\left(x^{-\frac{\lambda}{2}+\frac{1}{p}} G(x)\right) d x \leq J\|G\|_{q, \psi} .
$$

Then by (24) we have (23).

On the other hand, suppose that (23) is valid. Setting

$$
G(x):=y^{\frac{p \lambda}{2}-1}\left(\int_{0}^{\infty} k_{\lambda}^{(1)}(x y, 1) F_{\lambda}(x) d x\right)^{p-1} \quad\left(x \in \mathbf{R}_{+}\right)
$$

we find $\|G\|_{q, \psi}^{q}=J^{p}$. If $J=0$, then (24) is trivially valid; if $J=\infty$, then by (27) we have $\left\|F_{\lambda}\right\|_{p, \varphi}=\infty$, which contradicts the fact of (28). Assuming that $0<J<\infty$, then by (23) we 
have

$$
\begin{aligned}
& \|G\|_{q, \psi}^{q}=J^{p}=I<\prod_{s=1}^{2} k^{(s)}\left(\frac{\lambda}{2}\right)\|f\|_{p, \varphi}\|G\|_{q, \psi}, \\
& \|G\|_{q, \psi}^{q-1}=J<\prod_{s=1}^{2} k^{(s)}\left(\frac{\lambda}{2}\right)\|f\|_{p, \varphi},
\end{aligned}
$$

then we have (24), which is equivalent to (23).

Since we find similar to (28) that

$$
\begin{aligned}
\left\|G_{\lambda}\right\|_{q, \psi} & =\left[\int_{0}^{\infty} x^{q\left(1-\frac{\lambda}{2}\right)-1}\left(x^{\lambda-1} \int_{0}^{\infty} k_{\lambda}^{(3)}(x, y) g(y) d y\right)^{q} d x\right]^{\frac{1}{q}} \\
& =\left[\int_{0}^{\infty} x^{\frac{q \lambda}{2}-1}\left(\int_{0}^{\infty} k_{\lambda}^{(3)}(x, y) g(y) d y\right)^{q} d y\right]^{\frac{1}{q}}<k^{(3)}\left(\frac{\lambda}{2}\right)\|g\|_{q, \Psi},
\end{aligned}
$$

setting $G(x)=G_{\lambda}(x)$ in (23), we have (26).

For any $k>\max \left\{\frac{1}{|q| \delta_{1}}, \frac{1}{p \delta_{1}}\right\}(k \in \mathbf{N})$, we set

$$
\tilde{f}(x)=\left\{\begin{array}{ll}
x^{\frac{\lambda}{2}+\frac{1}{p k}-1}, & x \in(0,1), \\
0, & x \in[1, \infty),
\end{array} \quad \tilde{g}(y)= \begin{cases}0, & y \in(0,1], \\
y^{\frac{\lambda}{2}-\frac{1}{q k}-1}, & y \in(1, \infty)\end{cases}\right.
$$

Then we have

$$
\begin{aligned}
\widetilde{F}_{k}(y) & = \begin{cases}y^{\lambda-1} \int_{0}^{1} k_{\lambda}^{(2)}(x, y) x^{\frac{\lambda}{2}+\frac{1}{p k}-1} d x, & y \in(0,1), \\
0, & y \in[1, \infty)\end{cases} \\
& = \begin{cases}y^{\lambda-1} \int_{0}^{\infty} k_{\lambda}^{(2)}(x, y) \tilde{f}(x) d x, & y \in\{y>0 ; \widetilde{f}(y)>0\}, \\
0, & y \in\{y>0 ; \widetilde{f}(y)=0\},\end{cases} \\
\widetilde{G}_{k}(x) & := \begin{cases}x^{\lambda-1} \int_{1}^{\infty} k_{\lambda}^{(3)}(x, y) y^{\frac{\lambda}{2}-\frac{1}{q k}-1} d y, & x \in(1, \infty), \\
0, & x \in(0,1]\end{cases} \\
& = \begin{cases}x^{\lambda-1} \int_{0}^{\infty} k_{\lambda}^{(3)}(x, y) \widetilde{g}(y) d y, & x \in\{x>0 ; \widetilde{g}(x)>0\}, \\
0, & x \in\{x>0 ; \widetilde{g}(x)=0\} .\end{cases}
\end{aligned}
$$

If there exists a positive constant $K \leq \prod_{s=1}^{3} k^{(s)}\left(\frac{\lambda}{2}\right)$ such that (26) is valid when replacing $\prod_{s=1}^{3} k^{(s)}\left(\frac{\lambda}{2}\right)$ by $K$, then, in particular, we have

$$
\widetilde{L}_{k}=\frac{1}{k} \int_{0}^{\infty} \int_{0}^{\infty} k_{\lambda}^{(1)}(x y, 1) \widetilde{F}_{k}(y) \widetilde{G}_{k}(x) d y d x<\frac{1}{k} K\|\widetilde{f}\|_{p, \varphi}\|\widetilde{g}\|_{q, \psi}=K .
$$

By (17), we find $\prod_{s=1}^{3} k^{(s)}\left(\frac{\lambda}{2}\right)+o(1) \leq \widetilde{L}_{k}<K$, and then $\prod_{s=1}^{3} k^{(s)}\left(\frac{\lambda}{2}\right) \leq K(k \rightarrow \infty)$. Hence $K=\prod_{s=1}^{3} k^{(s)}\left(\frac{\lambda}{2}\right)$ is the best possible constant factor of (26).

The constant factor in (23) is the best possible. Otherwise, setting $G(x)=\widetilde{G}_{\lambda}(x)$, we would reach a contradiction that the constant factor in (26) is not the best possible. By the equivalency, if the constant factor in (24) is not the best possible, then by (29) we would reach a contradiction that the constant factor in (23) is not the best possible. 
Theorem 2 Suppose that $(1) \lambda \in A=(0, c)(0<c \leq \infty), k_{\lambda}^{(s)}(x, y)$ are non-negative homogeneous functions of degree $-\lambda$ in $\mathbf{R}_{+}^{2}$,

$$
k^{(s)}\left(\frac{\lambda}{2}\right)=\int_{0}^{\infty} k_{\lambda}^{(s)}(u, 1) u^{\frac{\lambda}{2}-1} d u \quad(s=1,2,3)
$$

there exists a constant $\delta_{0} \in\left(0, \frac{\lambda}{2}\right)$ such that $k^{(s)}\left(\frac{\lambda}{2} \pm \delta_{0}\right) \in \mathbf{R}_{+} ;(2) k_{\lambda}^{(2)}(u, 1)\left(k_{\lambda}^{(3)}(u, 1)\right)$ satisfies Condition (i) or Condition (ii); (3) if both $k_{\lambda}^{(2)}(u, 1)$ and $k_{\lambda}^{(3)}(u, 1)$ satisfy Condition (ii), then $k_{\lambda}^{(1)}(u, 1)$ satisfies Condition (iii); (3) if $k_{\lambda}^{(s)}(u, 1)(s=2,3)$ only satisfy Condition (i), then $\lambda \in$ $A$; otherwise, $\lambda \in(0,1) \cap A$. For $0<p<1, f(x), G(y) \geq 0, f \in L_{p, \varphi}\left(\mathbf{R}_{+}\right), G \in L_{q, \psi}\left(\mathbf{R}_{+}\right),\|f\|_{p, \varphi}$, $\|G\|_{q, \psi}>0$, and $F_{\lambda}(y)$ being as (22), we have the equivalent reverses of (24) and (25) with the best possible constant factor $\prod_{s=1}^{2} k^{(s)}\left(\frac{\lambda}{2}\right)$.

In particular, for $g(y) \geq 0, g \in L_{q, \psi}\left(\mathbf{R}_{+}\right),\|g\|_{q, \psi}>0$, and $G(x)=G_{\lambda}(x)$ as (25), we have the reverse of (26) with the best possible constant factor $\prod_{s=1}^{3} k^{(s)}\left(\frac{\lambda}{2}\right)$.

Proof By the reverse Hölder inequality (cf. [19]), we obtain the reverses of (27) and (28). Then we deduce the reverse of (24).

By the reverse Hölder inequality, we have

$$
I=\int_{0}^{\infty}\left(x^{\frac{\lambda}{2}-\frac{1}{p}} \int_{0}^{\infty} k_{\lambda}^{(1)}(x y, 1) F_{\lambda}(y) d y\right)\left(x^{-\frac{\lambda}{2}+\frac{1}{p}} G(x)\right) d x \geq J\|G\|_{q, \psi} .
$$

Then by the reverse of (24), we obtain the reverse of (23).

On the other hand, suppose that the reverse of (23) is valid. Setting $G(x)$ as (25), we find $\|G\|_{q, \psi}^{q}=J^{p}$. If $J=\infty$, then the reverse of (24) is trivially valid; if $J=0$, then by the reverse of (27), we have $\left\|F_{\lambda}\right\|_{p, \varphi}=0$, which contradicts the reverse of (28). Assuming that $0<J<\infty$, by the reverse of (23), we have

$$
\begin{aligned}
& \|G\|_{q, \psi}^{q}=J^{p}=I>\prod_{s=1}^{2} k^{(s)}\left(\frac{\lambda}{2}\right)\|f\|_{p, \varphi}\|G\|_{q, \psi}, \\
& \|G\|_{q, \psi}^{q-1}=J>\prod_{s=1}^{2} k^{(s)}\left(\frac{\lambda}{2}\right)\|f\|_{p, \varphi}
\end{aligned}
$$

and then the reverse of (24) follows, which is equivalent to the reverse of (23).

For $q<0$, since we find similar to the reverse of (28) that

$$
\begin{aligned}
\left\|G_{\lambda}\right\|_{q, \psi} & =\left[\int_{0}^{\infty} x^{q\left(1-\frac{\lambda}{2}\right)-1}\left(x^{\lambda-1} \int_{0}^{\infty} k_{\lambda}^{(3)}(x, y) g(y) d y\right)^{q} d x\right]^{\frac{1}{q}} \\
& =\left[\int_{0}^{\infty} x^{\frac{q \lambda}{2}-1}\left(\int_{0}^{\infty} k_{\lambda}^{(3)}(x, y) g(y) d y\right)^{q} d y\right]^{\frac{1}{q}}>k^{(3)}\left(\frac{\lambda}{2}\right)\|g\|_{q, \Psi},
\end{aligned}
$$

setting $G(x)=G_{\lambda}(x)$ in the reverse of (23), we have the reverse of (26).

For any $k>\max \left\{\frac{1}{|q| \delta_{\delta}}, \frac{1}{p \delta_{1}}\right\}(k \in \mathbf{N})$, we set $\widetilde{f}(x), \widetilde{g}(y)$ as Theorem 1 . If there exists a positive constant $K \geq \prod_{s=1}^{3} k^{(s)}\left(\frac{\lambda}{2}\right)$ such that the reverse of (26) is valid when replacing $\prod_{s=1}^{3} k^{(s)}\left(\frac{\lambda}{2}\right)$ 
by $K$, then, in particular, we have

$$
\widetilde{L}_{k}=\frac{1}{k} \int_{0}^{\infty} \int_{0}^{\infty} k_{\lambda}^{(1)}(x y, 1) \widetilde{F}_{k}(y) \widetilde{G}_{k}(x) d y d x>\frac{1}{k} K\|\widetilde{f}\|_{p, \varphi}\|\widetilde{g}\|_{q, \psi}=K .
$$

By (21), we find $\prod_{s=1}^{3} k^{(s)}\left(\frac{\lambda}{2}\right)+o(1)=\widetilde{L}_{k}>K$, and then $\prod_{s=1}^{3} k^{(s)}\left(\frac{\lambda}{2}\right) \geq K(k \rightarrow \infty)$. Hence $K=\prod_{s=1}^{3} k^{(s)}\left(\frac{\lambda}{2}\right)$ is the best possible constant factor of the reverse of (26).

The constant factor in the reverse of (23) is the best possible. Otherwise, setting $G(x)=$ $\widetilde{G}_{\lambda}(x)$, we would reach a contradiction that the constant factor in the reverse of (26) is not the best possible. By the equivalency, if the constant factor in the reverse of (24) is not the best possible, then by (30) we would reach a contradiction that the constant factor in the reverse of (23) is not the best possible.

\section{Some corollaries on Hilbert-Hardy-type inequalities}

In the following sections, if the best possible constant factor in a Hilbert-type inequality is related to $k_{j}^{(s)}\left(\frac{\lambda}{2}\right)(s=1,2,3, j=0,1,2)$ defined as follows, then we call this inequality Hilbert-Hardy-type inequality. The related operator is called Hilbert-Hardy-type operator.

Assuming that $k_{\lambda}^{(1)}(x y, 1)=0\left(0<\frac{1}{x} \leq y\right)$, we find $k_{\lambda}^{(1)}(u, 1)=0(u \geq 1)$, and

$$
k^{(1)}\left(\frac{\lambda}{2}\right)=k_{1}^{(1)}\left(\frac{\lambda}{2}\right):=\int_{0}^{1} k_{\lambda}^{(1)}(u, 1) u^{\frac{\lambda}{2}-1} d u
$$

By Theorem 1 and Theorem 2, we have the following.

Corollary 1 With the assumptions of Theorem 1 , for $p>1, k_{1}^{(1)}\left(\frac{\lambda}{2}\right) \in \mathbf{R}_{+}$, we have the following equivalent inequalities:

$$
\begin{aligned}
& \int_{0}^{\infty} G(x) \int_{0}^{\frac{1}{x}} k_{\lambda}^{(1)}(x y, 1) F_{\lambda}(y) d y d x<k_{1}^{(1)}\left(\frac{\lambda}{2}\right) k^{(2)}\left(\frac{\lambda}{2}\right)\|f\|_{p, \varphi}\|G\|_{q, \psi}, \\
& {\left[\int_{0}^{\infty} x^{\frac{p \lambda}{2}-1}\left(\int_{0}^{\frac{1}{x}} k_{\lambda}^{(1)}(x y, 1) F_{\lambda}(y) d y\right)^{p} d x\right]^{\frac{1}{p}}<k_{1}^{(1)}\left(\frac{\lambda}{2}\right) k^{(2)}\left(\frac{\lambda}{2}\right)\|f\|_{p, \varphi},}
\end{aligned}
$$

where the constant factor $k_{1}^{(1)}\left(\frac{\lambda}{2}\right) k^{(2)}\left(\frac{\lambda}{2}\right)$ is the best possible.

In particular, for $g(y) \geq 0, g \in L_{q, \psi}\left(\mathbf{R}_{+}\right),\|g\|_{q, \psi}>0, G(x)=G_{\lambda}(x)$ as (25), we have the following inequality:

$$
\int_{0}^{\infty} \int_{0}^{\frac{1}{\bar{x}}} k_{\lambda}^{(1)}(x y, 1) F_{\lambda}(y) G_{\lambda}(x) d y d x<k_{1}^{(1)}\left(\frac{\lambda}{2}\right) \prod_{s=2}^{3} k^{(s)}\left(\frac{\lambda}{2}\right)\|f\|_{p, \varphi}\|g\|_{q, \psi},
$$

where the constant factor $k_{1}^{(1)}\left(\frac{\lambda}{2}\right) \prod_{s=2}^{3} k^{(s)}\left(\frac{\lambda}{2}\right)$ is still the best possible.

Corollary 2 With the assumptions of Theorem 2 , for $0<p<1, k_{1}^{(1)}\left(\frac{\lambda}{2}\right) \in \mathbf{R}_{+}$, we have the equivalent reverses of (32) and (33), where the constant factor $k_{1}^{(1)}\left(\frac{\lambda}{2}\right) k^{(2)}\left(\frac{\lambda}{2}\right)$ is the best possible. 
In particular, for $g(y) \geq 0, g \in L_{q, \psi}\left(\mathbf{R}_{+}\right),\|g\|_{q, \psi}>0, G(x)=G_{\lambda}(x)$ as (25), we have the reverse of (34) with the best value $k_{1}^{(1)}\left(\frac{\lambda}{2}\right) \prod_{s=2}^{3} k^{(s)}\left(\frac{\lambda}{2}\right)$.

Assuming that $k_{\lambda}^{(1)}(x y, 1)=0\left(0<y \leq \frac{1}{x}\right)$, then we find $k_{\lambda}^{(1)}(u, 1)=0(0<u \leq 1)$, and

$$
k^{(1)}\left(\frac{\lambda}{2}\right)=k_{2}^{(1)}\left(\frac{\lambda}{2}\right):=\int_{1}^{\infty} k_{\lambda}^{(1)}(u, 1) u^{\frac{\lambda}{2}-1} d u .
$$

By Theorem 1 and Theorem 2, we have the following.

Corollary 3 With the assumptions of Theorem 1 , for $p>1, k_{2}^{(1)}\left(\frac{\lambda}{2}\right) \in \mathbf{R}_{+}$, we have the following equivalent inequalities:

$$
\begin{aligned}
& \int_{0}^{\infty} G(x) \int_{\frac{1}{x}}^{\infty} k_{\lambda}^{(1)}(x y, 1) F_{\lambda}(y) d y d x<k_{2}^{(1)}\left(\frac{\lambda}{2}\right) k^{(2)}\left(\frac{\lambda}{2}\right)\|f\|_{p, \varphi}\|G\|_{q, \psi}, \\
& {\left[\int_{0}^{\infty} x^{\frac{p \lambda}{2}-1}\left(\int_{\frac{1}{x}}^{\infty} k_{\lambda}^{(1)}(x y, 1) F_{\lambda}(y) d y\right)^{p} d x\right]^{\frac{1}{p}}<k_{2}^{(1)}\left(\frac{\lambda}{2}\right) k^{(2)}\left(\frac{\lambda}{2}\right)\|f\|_{p, \varphi},}
\end{aligned}
$$

where the constant factor $k_{2}^{(1)}\left(\frac{\lambda}{2}\right) k^{(2)}\left(\frac{\lambda}{2}\right)$ is the best possible.

In particular, for $g(y) \geq 0, g \in L_{q, \psi}\left(\mathbf{R}_{+}\right),\|g\|_{q, \psi}>0$, and $G(x)=G_{\lambda}(x)$ as (25), we have the following inequality:

$$
\int_{0}^{\infty} G_{\lambda}(x) \int_{\frac{1}{\bar{x}}}^{\infty} k_{\lambda}^{(1)}(x y, 1) F_{\lambda}(y) d y d x<k_{2}^{(1)}\left(\frac{\lambda}{2}\right) \prod_{s=2}^{3} k^{(s)}\left(\frac{\lambda}{2}\right)\|f\|_{p, \varphi}\|g\|_{q, \psi},
$$

where the constant factor $k_{2}^{(1)}\left(\frac{\lambda}{2}\right) \prod_{s=2}^{3} k^{(s)}\left(\frac{\lambda}{2}\right)$ is still the best possible.

Corollary 4 With the assumptions of Theorem 2, if $0<p<1, k_{2}^{(1)}\left(\frac{\lambda}{2}\right) \in \mathbf{R}_{+}$, we have the equivalent reverses of (36) and (37), where the constant factor $k_{2}^{(1)}\left(\frac{\lambda}{2}\right) k^{(2)}\left(\frac{\lambda}{2}\right)$ is the best possible.

In particular, for $g(y) \geq 0, g \in L_{q, \psi}\left(\mathbf{R}_{+}\right),\|g\|_{q, \psi}>0$, and $G(x)=G_{\lambda}(x)$ as (25), we have the reverse of (38) with the best value $k_{2}^{(1)}\left(\frac{\lambda}{2}\right) \prod_{s=2}^{3} k^{(s)}\left(\frac{\lambda}{2}\right)$.

Remark 3 For $x>0$, we set $A_{x, 0}:=(0, \infty), A_{x, 1}:=\left(0, \frac{1}{x}\right), A_{x, 2}:=\left(\frac{1}{x}, \infty\right)$. By (24), (33) and (37), putting $k_{0}^{(1)}\left(\frac{\lambda}{2}\right):=k^{(1)}\left(\frac{\lambda}{2}\right)$, for $i=0,1,2$, we have the following Hilbert-Hardy-type inequalities:

$$
\left[\int_{0}^{\infty} x^{\frac{p \lambda}{2}-1}\left(\int_{A_{x, i}} k_{\lambda}^{(1)}(x y, 1) F_{\lambda}(y) d y\right)^{p} d x\right]^{\frac{1}{p}}<k_{i}^{(1)}\left(\frac{\lambda}{2}\right) k^{(2)}\left(\frac{\lambda}{2}\right)\|f\|_{p, \varphi},
$$

where the constant factor $k_{i}^{(1)}\left(\frac{\lambda}{2}\right) k^{(2)}\left(\frac{\lambda}{2}\right)(i=0,1,2)$ is still the best possible.

For $x>0$, we set some sets $B_{x, 0}:=(0, \infty), B_{x, 1}:=(x, \infty), B_{x, 2}:=(0, x)$. If $k_{\lambda}^{(2)}(x, y)=0(y \in$ $\left.\mathbf{R}_{+} \backslash B_{x, 1}\right)$, then we find $k_{\lambda}^{(2)}(u, 1)=0(u \geq 1)$, and

$$
k^{(2)}\left(\frac{\lambda}{2}\right)=k_{1}^{(2)}\left(\frac{\lambda}{2}\right):=\int_{0}^{1} k_{\lambda}^{(2)}(u, 1) u^{\frac{\lambda}{2}-1} d u ;
$$


if $k_{\lambda}^{(2)}(x, y)=0\left(y \in \mathbf{R}_{+} \backslash B_{x, 2}\right)$, then we find $k_{\lambda}^{(2)}(u, 1)=0(0<u \leq 1)$, and

$$
k^{(2)}\left(\frac{\lambda}{2}\right)=k_{2}^{(2)}\left(\frac{\lambda}{2}\right):=\int_{1}^{\infty} k_{\lambda}^{(2)}(u, 1) u^{\frac{\lambda}{2}-1} d u
$$

Assuming that $k_{0}^{(2)}\left(\frac{\lambda}{2}\right):=k^{(2)}\left(\frac{\lambda}{2}\right), k_{i}^{(1)}\left(\frac{\lambda}{2}\right), k_{j}^{(2)}\left(\frac{\lambda}{2}\right) \in \mathbf{R}_{+}$, for $i, j=0,1,2$, setting

$$
F_{\lambda, j}(y):= \begin{cases}y^{\lambda-1} \int_{B_{x, j}} k_{\lambda}^{(2)}(x, y) f(x) d x, & y \in\left\{y \in \mathbf{R}_{+} ; f(y)>0\right\}, \\ 0, & y \in\left\{y \in \mathbf{R}_{+} ; f(y)=0\right\},\end{cases}
$$

then it follows that $F_{\lambda, 0}(y)=F_{\lambda}(y)$, and by (39) we have the following united expression of Hilbert-Hardy-type inequalities:

$$
\left[\int_{0}^{\infty} x^{\frac{p \lambda}{2}-1}\left(\int_{A_{x, i}} k_{\lambda}^{(1)}(x y, 1) F_{\lambda, j}(y) d y\right)^{p} d x\right]^{\frac{1}{p}}<k_{i}^{(1)}\left(\frac{\lambda}{2}\right) k_{j}^{(2)}\left(\frac{\lambda}{2}\right)\|f\|_{p, \varphi},
$$

where the constant factor $k_{i}^{(1)}\left(\frac{\lambda}{2}\right) k_{j}^{(2)}\left(\frac{\lambda}{2}\right)(i, j=0,1,2)$ is the best possible.

In the same way, we still can find by (27) and (28) that

$$
\begin{aligned}
& {\left[\int_{0}^{\infty} x^{\frac{p \lambda}{2}-1}\left(\int_{A_{x, i}} k_{\lambda}^{(1)}(x y, 1) F_{\lambda, j}(y) d y\right)^{p} d x\right]^{\frac{1}{p}} \leq k_{i}^{(1)}\left(\frac{\lambda}{2}\right)\left\|F_{\lambda, j}\right\|_{p, \varphi},} \\
& \left\|F_{\lambda, j}\right\|_{p, \varphi}<k_{j}^{(2)}\left(\frac{\lambda}{2}\right)\|f\|_{p, \varphi} \quad(i, j=0,1,2),
\end{aligned}
$$

where the constant factors $k_{i}^{(1)}\left(\frac{\lambda}{2}\right)$ and $k_{j}^{(2)}\left(\frac{\lambda}{2}\right)$ are the best possible.

Example 2 (i) For $k_{\lambda}^{(s)}(x, y)=\frac{\left.|\ln x / y|\right|^{\beta-1}}{(\max \{x, y\})^{\lambda}}(\lambda>0, \beta \geq 1 ; s=1,2,3)$, we find

$$
\begin{array}{rl}
k_{0}^{(s)}\left(\frac{\lambda}{2}\right) & =\int_{0}^{\infty} k_{\lambda}^{(s)}(u, 1) u^{\frac{\lambda}{2}-1} d u=\int_{0}^{\infty} \frac{|\ln u|^{\beta-1}}{(\max \{u, 1\})^{\lambda}} u^{\frac{\lambda}{2}-1} d u \\
& =2 \int_{0}^{1}(-\ln u)^{\beta-1} u^{\frac{\lambda}{2}-1} d u \\
\stackrel{v}{v}=-\ln u & 2 \int_{0}^{\infty} e^{-\frac{\lambda}{2} v} v^{\beta-1} d v=2\left(\frac{2}{\lambda}\right)^{\beta} \Gamma(\beta), \\
k_{i}^{(s)}\left(\frac{\lambda}{2}\right) & =\left(\frac{2}{\lambda}\right)^{\beta} \Gamma(\beta) \quad(i=1,2) .
\end{array}
$$

(ii) For $k_{\lambda}^{(s)}(x, y)=\frac{1}{|x-y|^{\lambda}}(0<\lambda<1 ; s=1,2,3)$, we find

$$
\begin{aligned}
k_{0}^{(s)}\left(\frac{\lambda}{2}\right) & =\int_{0}^{\infty} k_{\lambda}^{(s)}(u, 1) u^{\frac{\lambda}{2}-1} d u=\int_{0}^{\infty} \frac{1}{|u-1|^{\lambda}} u^{\frac{\lambda}{2}-1} d u \\
& =2 \int_{0}^{1} \frac{1}{(1-u)^{\lambda}} u^{\frac{\lambda}{2}-1} d u=2 B\left(1-\lambda, \frac{\lambda}{2}\right), \\
k_{i}^{(s)}\left(\frac{\lambda}{2}\right) & =B\left(1-\lambda, \frac{\lambda}{2}\right) \quad(i=1,2) .
\end{aligned}
$$




\section{A composition of two Hilbert-Hardy-type operators}

For $F \in L_{p, \varphi}\left(\mathbf{R}_{+}\right)$, we set $h_{i}(x):=x^{\lambda-1} \int_{A_{x, i}} k_{\lambda}^{(1)}(x y, 1) F(y) d y\left(x \in \mathbf{R}_{+} ; i=0,1,2\right)$. Then by (41) we have

$$
\left\|h_{i}\right\|_{p, \varphi} \leq k_{i}^{(1)}\left(\frac{\lambda}{2}\right)\|F\|_{p, \varphi} .
$$

Definition 3 With the assumptions of Theorem 1 , for any $i=0,1,2, k_{i}^{(1)}\left(\frac{\lambda}{2}\right) \in \mathbf{R}_{+}$, we define a Hilbert-Hardy-type operator $T_{1}^{(i)}: L_{p, \varphi}\left(\mathbf{R}_{+}\right) \rightarrow L_{p, \varphi}\left(\mathbf{R}_{+}\right)$as follows: For any $F \in L_{p, \varphi}\left(\mathbf{R}_{+}\right)$, there exists a unified expression $T_{1}^{(i)} F=h_{i} \in L_{p, \varphi}\left(\mathbf{R}_{+}\right)$such that for any $x \in \mathbf{R}_{+}, T_{1}^{(i)} F(x)=$ $h_{i}(x)$.

By (43), we have $\left\|T_{1}^{(i)} F\right\|_{p, \varphi} \leq k_{i}^{(1)}\left(\frac{\lambda}{2}\right)\|F\|_{p, \varphi}$. Hence, $T_{1}^{(i)}$ is a bounded linear operator with

$$
\left\|T_{1}^{(i)}\right\|:=\sup _{F(\not \theta) \in L_{p, \varphi}\left(\mathbf{R}_{+}\right)} \frac{\left\|T_{1}^{(i)} F\right\|_{p, \varphi}}{\|F\|_{p, \varphi}} \leq k_{i}^{(1)}\left(\frac{\lambda}{2}\right) .
$$

Since the constant factor in (43) is the best possible, we have $\left\|T_{1}^{(i)}\right\|=k_{i}^{(1)}\left(\frac{\lambda}{2}\right)$.

Definition 4 With the assumptions of Theorem 1 , for any $j=0,1,2, k_{j}^{(2)}\left(\frac{\lambda}{2}\right) \in \mathbf{R}_{+}$, we define a Hilbert-Hardy-type operator $T_{2}^{(j)}: L_{p, \varphi}\left(\mathbf{R}_{+}\right) \rightarrow L_{p, \varphi}\left(\mathbf{R}_{+}\right)$as follows: For any $f \in L_{p, \varphi}\left(\mathbf{R}_{+}\right)$, there exists a unified expression $T_{2}^{(j)} f=F_{\lambda, j} \in L_{p, \varphi}\left(\mathbf{R}_{+}\right)$such that for any $y \in \mathbf{R}_{+}, T_{2}^{(j)} f(y)=$ $F_{\lambda, j}(y)$.

By (42), we have $\left\|T_{2}^{(j)} f\right\|_{p, \varphi}=\left\|F_{\lambda, j}\right\|_{p, \varphi} \leq k_{j}^{(2)}\left(\frac{\lambda}{2}\right)\|f\|_{p, \varphi}$. Hence, $T_{2}^{(j)}$ is a bounded linear operator with

$$
\left\|T_{2}^{(j)}\right\|=\sup _{f(\nexists \theta) \in L_{p, \varphi}\left(\mathbf{R}_{+}\right)} \frac{\left\|T_{2}^{(j)} f\right\|_{p, \varphi}}{\|f\|_{p, \varphi}} \leq k_{j}^{(2)}\left(\frac{\lambda}{2}\right) .
$$

Since the constant in (42) is the best possible, we have $\left\|T_{2}^{(j)}\right\|=k_{j}^{(2)}\left(\frac{\lambda}{2}\right)$.

Definition 5 With the assumptions of Theorem 1, for any $i, j \in\{0,1,2\}, k_{i}^{(1)}\left(\frac{\lambda}{2}\right), k_{j}^{(2)}\left(\frac{\lambda}{2}\right) \in$ $\mathbf{R}_{+}$, we define a Hilbert-Hardy-type operator $T_{i, j}: L_{p, \varphi}\left(\mathbf{R}_{+}\right) \rightarrow L_{p, \varphi}\left(\mathbf{R}_{+}\right)$as follows: For any $f \in L_{p, \varphi}\left(\mathbf{R}_{+}\right)$, there exists a unified expression $T_{i, j} f=T_{1}^{(i)} F_{\lambda, j} \in L_{p, \varphi}\left(\mathbf{R}_{+}\right)$such that for any $x \in \mathbf{R}_{+}$,

$$
T_{i, j} f(x)=T_{1}^{(i)} F_{\lambda, j}(x)=x^{\lambda-1} \int_{A_{x, i}} k_{\lambda}^{(1)}(x y, 1) F_{\lambda, j}(y) d y .
$$

It is evident that $T_{i, j} f=T_{1}^{(i)} F_{\lambda, j}=T_{1}^{(i)}\left(T_{2}^{(j)} f\right)=\left(T_{1}^{(i)} T_{2}^{(j)}\right) f$, and then $T_{i, j}=T_{1}^{(i)} T_{2}^{(j)}$. Hence, $T_{i, j}$ is the composition of $T_{1}^{(i)}$ and $T_{2}^{(j)}$, and (cf. [20])

$$
\left\|T_{i, j}\right\|=\left\|T_{1}^{(i)} T_{2}^{(j)}\right\| \leq\left\|T_{1}^{(i)}\right\| \cdot\left\|T_{2}^{(j)}\right\|=k_{i}^{(1)}\left(\frac{\lambda}{2}\right) k_{j}^{(2)}\left(\frac{\lambda}{2}\right) .
$$

By (40), we have

$$
\left\|T_{i, j} f\right\|_{p, \varphi}=\left\|T_{1}^{(i)} F_{\lambda, j}\right\|_{p, \varphi} \leq k_{i}^{(1)}\left(\frac{\lambda}{2}\right) k_{j}^{(2)}\left(\frac{\lambda}{2}\right)\|f\|_{p, \varphi} .
$$

Since the constant factor in (40) is the best possible, then the theorem follows. 
Theorem 3 With the assumptions of Theorem 1 , if for any $i, j \in\{0,1,2\}, k_{i}^{(1)}\left(\frac{\lambda}{2}\right), k_{j}^{(2)}\left(\frac{\lambda}{2}\right) \in$ $\mathbf{R}_{+}$, then we have the composition formula of two Hilbert-Hardy-type operators as follows:

$$
\left\|T_{i, j}\right\|=\left\|T_{1}^{(i)} T_{2}^{(j)}\right\|=\left\|T_{1}^{(i)}\right\| \cdot\left\|T_{2}^{(j)}\right\|=k_{i}^{(1)}\left(\frac{\lambda}{2}\right) k_{j}^{(2)}\left(\frac{\lambda}{2}\right) .
$$

Example 3 For $k_{\lambda}^{(1)}(x y, 1)=\frac{1}{|x y-1|^{\lambda}}, k_{\lambda}^{(2)}(x, y)=\frac{|\ln (x / y)|^{\beta-1}}{(\max \{x, y\})^{\lambda}}(\beta \geq 1), \lambda \in(0,1)$, by Example 2 and (44), we have

$$
\begin{aligned}
& \left\|T_{0,0}\right\|=\left\|T_{1}^{(0)} T_{2}^{(0)}\right\|=\left\|T_{1}^{(0)}\right\| \cdot\left\|T_{2}^{(0)}\right\|=4 B\left(1-\lambda, \frac{\lambda}{2}\right)\left(\frac{2}{\lambda}\right)^{\beta} \Gamma(\beta), \\
& \left\|T_{0, j}\right\|=\left\|T_{1}^{(0)} T_{2}^{(j)}\right\|=\left\|T_{1}^{(0)}\right\| \cdot\left\|T_{2}^{(j)}\right\|=2 B\left(1-\lambda, \frac{\lambda}{2}\right)\left(\frac{2}{\lambda}\right)^{\beta} \Gamma(\beta) \quad(j=1,2), \\
& \left\|T_{i, 0}\right\|=\left\|T_{1}^{(i)} T_{2}^{(0)}\right\|=\left\|T_{1}^{(i)}\right\| \cdot\left\|T_{2}^{(0)}\right\|=2 B\left(1-\lambda, \frac{\lambda}{2}\right)\left(\frac{2}{\lambda}\right)^{\beta} \Gamma(\beta) \quad(i=1,2), \\
& \left\|T_{i, j}\right\|=\left\|T_{1}^{(i)} T_{2}^{(j)}\right\|=\left\|T_{1}^{(i)}\right\| \cdot\left\|T_{2}^{(j)}\right\|=B\left(1-\lambda, \frac{\lambda}{2}\right)\left(\frac{2}{\lambda}\right)^{\beta} \Gamma(\beta) \quad(i, j=1,2) .
\end{aligned}
$$

\section{Competing interests}

The authors declare that they have no competing interests.

\section{Authors' contributions}

BY carried out the mathematical studies, participated in the sequence alignment and drafted the manuscript. QC participated in the design of the study and performed the numerical analysis. All authors read and approved the final manuscript.

\section{Author details}

'Department of Mathematics, Guangdong University of Education, Guangzhou, Guangdong 510303, P.R. China.

${ }^{2}$ Department of Computer Science, Guangdong University of Education, Guangzhou, Guangdong 510303, P.R. China.

\section{Acknowledgements}

This work is supported by the National Natural Science Foundation of China (No. 61370186), and 2013 Knowledge Construction Special Foundation Item of Guangdong Institution of Higher Learning College and University (No. 2013KJCX0140).

\section{Received: 26 October 2014 Accepted: 5 March 2015 Published online: 18 March 2015}

\section{References}

1. Schur, l: Bernerkungen zur theorie der beschränkten bilinearformen mit unendlich vielen veränderlichen. J. Math. 140, 1-28 (1911)

2. Hardy, GH: Note on a theorem of Hilbert concerning series of positive term. Proc. Lond. Math. Soc. 23, 45-46 (1925)

3. Hardy, GH, Littlewood, JE, Pólya, G: Inequalities. Cambridge University Press, Cambridge (1934)

4. Mitrinović, DS, Pečarić, JE, Fink, AM: Inequalities Involving Functions and Their Integrals and Derivatives. Mathematics and Its Applications, vol. 53. Kluwer Academic, Boston (1991)

5. Yang, B: On Hilbert's integral inequality. J. Math. Anal. Appl. 220, 778-785 (1998)

6. Yang, B: The Norm of Operator and Hilbert-Type Inequalities. Science Press, Beijing (2009)

7. Carleman, T: Sur les équations intégrales singulieres à noyau réel et symétrique. Lundequistska Bokhandeln, Uppsala (1923)

8. Wilhelm, M: On the spectrum of Hilbert's inequality. Am. J. Math. 72, 699-704 (1950)

9. Zhang, KW: A bilinear inequality. J. Math. Anal. Appl. 271, 288-296 (2002)

10. Yang, B: On the norm of an integral operator and applications. J. Math. Anal. Appl. 321, 182-192 (2006)

11. Arpad, B, Choonghong, O: Best constant for certain multilinear integral operator. J. Inequal. Appl. 2006, Article ID 28582 (2006)

12. Huang, Q, Yang, B: On a multiple Hilbert-type integral operator and applications. J. Inequal. Appl. 2009, Article ID 192197 (2009)

13. Yang, B, Xie, M: A Hilbert-Hardy-type inequality. Appl. Math. Sci. 6(67), 3321-3327 (2012)

14. Adiyasuren, V, Batbold, T, Krnić, M: On several new Hilbert-type inequalities involving means operators. Acta Math. Sin. Engl. Ser. 29(8), 1493-1514 (2013)

15. Liu, X, Yang, B: On a new Hilbert-Hardy-type integral operator and applications. J. Inequal. Appl. 2010, Article ID 812636 (2010) 
16. Adiyasuren, V, Batbold, T, Krnić, M: Half-discrete Hilbert-type inequalities with mean operators, the best constants, and applications. Appl. Math. Comput. 231, 148-159 (2014)

17. Yang, B: Hillbert-Type Integral Inequalities. Bentham Science Publishers, Sharjah (2009)

18. Kuang, J: Introduction to Real Analysis. Hunan Education Press, Changsha (1996)

19. Kuang, J: Applied Inequalities. Shangdong Science and Technology Press, Jinan (2004)

20. Taylor, AE, Lay, DC: Introduction to Functional Analysis. Wiley, New York (1980)

Submit your manuscript to a SpringerOpen ${ }^{\circ}$ journal and benefit from:

- Convenient online submission

- Rigorous peer review

- Immediate publication on acceptance

- Open access: articles freely available online

- High visibility within the field

- Retaining the copyright to your article 